\title{
Development of Maturity Levels for Agile Industrial Ser- vice Companies
}

\author{
Achim Kampker ${ }^{1}$, Jana Frank ${ }^{1}$; Roman Emonts-Holley ${ }^{1}$, Philipp Jussen ${ }^{1}$ \\ ${ }^{1}$ Resarch Institute for Operations Management (FIR) at RWTH Aachen University, \\ Campus-Boulevard 55, 52074 Aachen
}

\begin{abstract}
Industrial service is currently undergoing tremendous changes, largely driven by the development of new technologies, in particular the advancing digitalization. Never before have organizations had more comprehensive and insightful data assets - and never before have the opportunities to fully exploit this potential been better. However, most companies are unaware of how they can make use of this potential and which development steps are necessary to react to the current situation. To change this, a maturity-based approach was developed which describes four development stages of an industrial service company from a technological, organizational and cultural point of view. The maturity model makes it possible to develop a digital roadmap that is tailor-made to each company, which helps to introduce Industrie 4.0 and transform industrial service companies into learning, agile organizations.
\end{abstract}

Keywords: Industrie 4.0, maturity model, technical service, service management, culture, organizational structure.

\section{Introduction}

Recent developments in ICT (information and communication technologies) cause highly impactful changes in the industrial services sector. The digitization of service systems makes it possible to integrate ICT in different areas of a company, from sales and service provision to the development of entirely new services [1]. New technologies play a key role as tools for exploiting the changes brought about by digitization and Industrie 4.0 as an opportunity for industrial service. Networked, intelligent machines and plants cannot only control the production process automatically, but also schedule maintenance services and repairs by monitoring their own condition. With the help of remote technologies, it is also possible to undertake maintenance measures from a distance anytime. These and many other practical applications of ICT are just the beginning of an extensive revolution in the service sector [2]. The use of new technologies and the acquisition of knowledge from a target-oriented information processing change the distribution of responsibilities and working methods of companies significantly. New technologies are being introduced, but very few organizations did further changes, such as a fundamental structural and process reform to integrate new technologies to raise the full potential. [3]. In the course of digitalization, organizational and cultural areas of a company must also be transformed. Although modern technologies make it possible to build an ever-growing database, harnessing the underlying potential depends just as much as on the organizational structure and culture in the company. For 
a service company, the overall objective should be to build a learning, agile service organization that can continuously adapt to a dynamic environment. For this purpose, traditional, hierarchical company structures and values need to be loosened. Nearly 60 percent of large companies are still traditionally managed - more than twice as many as medium-sized companies [4]. A simple reorganization is unlikely to suffice in most cases. Contemporary corporate identity precepts such as feedback orientation trust or fault tolerance can hardly be found among service companies [5].

New technologies in the industrial service sector also change the demands made on the company organization and corporate culture. The transfer of decision-making power to digital systems, the trust in data provided by these systems as well as the sharing of knowledge become crucial factors of success. The introduction of new technologies makes it possible to realize potentials in service, but only if employees accept these technologies, incorporate knowledge into systems and trust decision templates from systems. These technologies must be integrated efficiently into the existing organizational structure. On these grounds, a maturity model for industrial service was developed, which describes the development stages of a company from a technological, organizational and cultural point of view. It is constructed as a four-stage maturity model, whereby benefits for the company increase with each stage. The maturity model makes it possible to develop a digital roadmap, tailor-made to each company, which helps to introduce Industrie 4.0 and transform industrial service companies into a agile organization capable and willing to learn.

\section{Fundamental Definitions and State of Research}

The degree of maturity describes the maturity of a field of observation with regard to a specific method or a management model. Changing degrees of consistency between defined criteria and degrees of fulfillment of these criteria result in different levels of maturity. [6] So far, there are a variety of maturity models that assess the current state of digitalization and Industrie 4.0 in companies, such as the so-called acatech Industrie 4.0 Maturity Index. It is a standardized and well-established approach for the assessment of a company's status during its transformation towards becoming an agile company. The company is evaluated from technological, resource-related, organizational and cultural perspectives [7]. The maturity model is divided into six levels: computerization, connectivity, visibility, transparency, predictability and adaptability [7]. The acatech model refers primarily to manufacturing companies and not to service providers, which is why it does not give any indication of how industrial service is changing.

In the field of industrial service, there are primarily maturity models which describe the development stages of a company with regard to the service and spare parts portfolio, the professionalism and effectiveness of the organizational structure and the prevailing service processes $[8,9]$. A source that considers all fields does not exist yet. Thus, this work contributes to close the research gab between Industrie 4.0 in the service sector and technological change and its impact on the structure and culture of a service company. 


\section{$3 \quad$ Methodology}

The close cooperation with the project partners and the chosen practical methodological approach is carried out as a combination of a workshop and in-depth literature review. The described acatech maturity index was used as a framework for the design of the maturity degrees. As mentioned before, it refers primarily to manufacturing companies and therefore does not give any indication of how industrial service is changing.

20 representatives of the German maintenance industry took part in the workshop. In a first step, they were asked to describe their current technologies. Second, they were asked to discuss relevant future technologies, some of which are already being planned. Based on these results, it is possible to estimate which maintenance technologies are relevant in the near future. In a third step, the participants discussed about organizational changes through the introduction of new technologies by first agreeing on a short definition and delimitation of the two terms company culture and organizational structure. The result was a description of the organizational and cultural framework conditions under which technological developments in service can take place successfully. As a follow-up task to the workshop, the respective technologies as well as the framework conditions were assigned to the different levels of maturity, resulting in a maturity model for the industrial service, which describes four development steps towards an agile, learning service organization. The development stages are visibility, transparency, predictability and adaptability. They have been described in a holistic approach with regard to technology, organization and culture.

\section{Industrial Service Maturity Model}

The maturity level description is based on the different levels of technology development. Figure 1 provides an overview of the above-mentioned target variables.
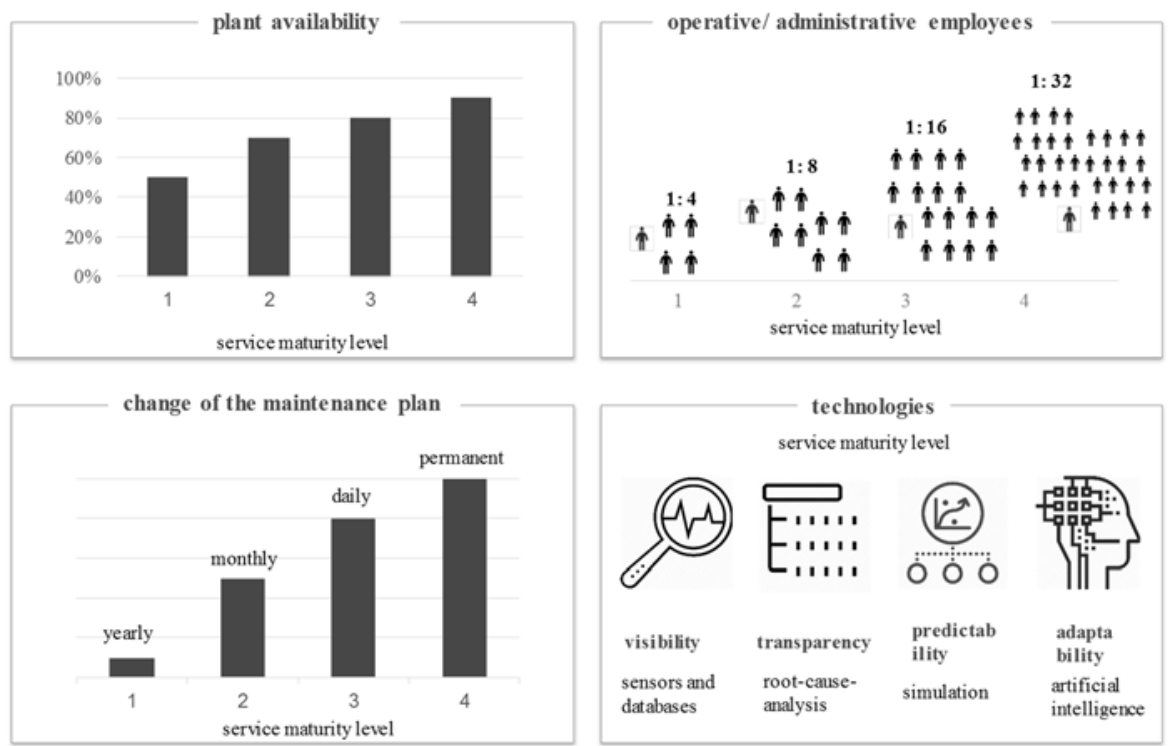

Fig. 1. Parameters of the maturity levels in industrial service 
As shown in figure 1, the use of preventive maintenance measures increased the availability of plants significantly as less reactive and more preventive maintenance is used. This causes a reduction in unplanned downtime, which cannot be avoided entirely though, as stage four shows - despite full automation. Based on available data, the maintenance plans may be subject to permanent change. The complexity of the interaction between production orders and maintenance requirements results in a system that enables constant recalculation of scenarios and simulations starting at level three. At the same time, decisions about the levels are increasingly taken over by systems, which in turn means that fewer and fewer employees are needed for administrative activities.

In the following, the four maturity levels are described in detail. All descriptions follow the same scheme by first giving a brief overview of the technology of the stage and in the second step describing the maturity of the organization and culture based on the technological implications. For the organization, only the dimension decision management is considered. For company culture, the aspect of decision processes is described in an analogue way.

\subsection{Visibility}

Regarding the level of visibility, a condition monitoring system is the predominant technology. Data provides a complete picture of what is currently happening. Sensor, process and order data is read and manually evaluated in order to create a so-called digital twin of the machine, its mapping order status and the resources used. At this stage, only reactive or preventive maintenance measures are possible. They are based on wear and tear details or runtime-dependent intervals.

\section{Technology}

Machine data:

Vibration analysis, temperature data, performance data (voltage etc.), indirect performance data (lubricant, residual stress of the material). This data can be viewed in real-time via a dashboard and is also available historically via drill-down. Error messages are displayed in plain text to the machine operator and service provider. Based on an error message, the service provider can be commissioned. Depending on the contract, the service provider reports an error message directly to the machine operator.

\section{Order data:}

Orders are generated and scheduled as a preventive measure on the basis of machine data and manufacturer specifications. The planner of the service agent must schedule the orders manually. Each order contains all relevant order details: contact data, object data and object list, usage history, documents, material reservations and notifications. The customer can check the order status (in progress, planned, executed, etc.) in real time. The technician enters the following information in the system: time confirmation (directions, working time), material confirmation and service report.

\section{Organization}

Decisions can be made quickly and easily thanks to a sufficient data transparency. For the organization, this means that decisions must be based on data and hierarchies 
and release procedures become superfluous. This is problematic for companies with contractual liability. Managers must be responsible for decisions in order to ensure liability. At this point, contractual framework conditions must be created to secure decisions made by employees.

\section{Culture}

The employees base decisions and discussions on data. In doing so, Data-based decisions can be considered as having a higher value than personal opinions. Employees have to come to terms with the fact that everything is recorded in form of data. They also have to learn to trust this data and, at the same time, to question it critically.

\subsection{Transparency}

This level is characterized by root-cause-analysis, decision trees, data- and advanced analytics. In the case of the latter, the stored and aggregated data for the analysis of error causes is interlinked. Big Data is refined to so-called Smart Data. Technologically, the method of Edge Computing is used for this. Edge computing enables an acceleration of data streams, e.g. real-time data processing without latency times. This enables intelligent applications and devices to react to data almost immediately when it is created. This avoids delays. The systems are self-learning and present the user with a simple and intuitive way to combine different data sets. Data scientists and machine learning algorithms are indispensable for this task. The difference of this method to heuristic ones is the run-up time. Training a neural network requires great effort in the beginning. Once in operation, the system can deliver results in real-time. Heuristic methods require very little effort in the run-up phase, but can only achieve a result by running many calculations. This means that a long runtime can be expected for each request.

\section{Technology}

\section{Machine data:}

The machine data described for the visibility level is automatically analyzed in the transparency level. Historical data of the machine is used for this purpose in order to automatically uncover correlations between the causes of faults. The failure of the bearing of an electric motor, for example, is a result of a combination of oil pressure, residual stress and torque. This conclusion was derived from historical data and checked by employees for meaningfulness.

\section{Order data:}

Orders can be evaluated historically by the system, i.e. the necessity and time of the order can be automatically classified on a scale covering the options "optimal, too early, and too late". For this purpose, the performance data of the machine is compared before and after a maintenance service has been performed.

\section{Organization}

At this maturity level, human-machine interaction is of importance. The existing expert knowledge of the maintenance technician must be transferred to the system. The 
team structure of the operative unit changes due to the increase of the number of employees who may not have manual skills but possess mathematical-analytical skills. Teams must be able to get together quickly and work together locally in consideration of their competencies and the task to be solved. Decisions must be implemented extremely quickly, which affects hierarchical structures and release processes.

\section{Culture}

Employee knowledge influences the design of analysis models. This requires a willingness to share knowledge with the system and the understanding of the analysis models. The analysis models allow the detection of contexts, so that employees can draw conclusions. Information as the basis for decisions are available in a simpler form. Decisions can be made faster. Employees need to learn how to handle these faster available data-driven decisions.

\subsection{Predictability}

The forecasting ability level is characterized by the forecasting of events and predictive maintenance. Systems can predict scenarios with probabilities and provide them to the user. The user needs to evaluate them and then react accordingly. Historical data can be combined and evaluated and used for future predictions. For this purpose, forecasting methods are used that can analyze dynamic non-linear relationships between numerous parameters and, based on these, make forecasts for the future within a suitable period of time. The machines and systems are maintained proactively to minimize downtimes. Ideally, faults can be predicted before defects or failures occur. The actual occurrence of the fault can be prevented by the maintenance measures that are proactively initiated at an early stage. For reliable predictions regarding the condition of machines, systems and faults, it is necessary to collect large amounts of data. In addition to data from the machines, data from peripheral and environmental characteristics such as temperature or humidity levels is also collected. The actual result of the scenario must be closely observed and feed back into the system. This guarantees better forecasts. There is great potential for this maturity level: profitability increases through predictive maintenance because fewer reactive and preventive orders need to be carried out. As a result, machine downtime is reduced dramatically.

\section{Technology}

Machine data.

The system records the status of the machine and can simulate various scenarios based on the planned orders. Thus, the system knows different future states of the machine. Recommendations for the optimal handling of the machine (on the part of production and service) can be issued by the system. Based on the objectives of the company, the service provider and the machine operator can select the right configuration. The current status of the machines or system components is displayed on a dashboard. Forecasting technology, in turn, creates risk profiles as to when and what disruption is to be expected. The forecast reports show in detail when which phases begin and end, i.e. when the condition of the machine becomes critical. In this way, it is possible to determine when the time window during which maintenance should be carried out 
opens and closes in order to prevent unplanned breakdowns as far as possible and the same time make optimum use of remaining service lives.

Order data.

Based on the created risk profiles and the forecast of when a plant condition will become critical, orders can be planned much better. This means that maintenance orders are planned based on the selected periods or scenarios. Periods refer to the lead time, which is determined according to the criticality of the investment. For a bottleneck machine, for example, the calculated worst-case scenario is used, while the best-case scenario is selected for non-critical facilities. It is the responsibility of the customer and the scheduler to make these decisions. To this end, rules and frameworks should be defined in which the procedure and the systemic limits are shown and defined.

\section{Organization}

In this stage, the worker uses the expert systems that were filled based on the employee's knowledge. Decisions are thus prepared based on data and made available to the employee. Extreme flexibility of maintenance staff is required, as orders can change daily. Significantly greater competence and understanding of the planning content is required when setting up the system. Continuous testing of the scenarios at the beginning is necessary to determine the system limits.

\section{Culture}

The selection of a course of action and therefore the preparation of decisions is transferred to the machine. The work is no longer based on the knowledge and experience of the workers, but on the information and learning ability of the machine. However, willingness to cooperate requires trust in data and technology.

\subsection{Adaptability}

At this stage, decisions are made by the system. For this purpose, the data of the digital shadow is used in such a way that decisions with the greatest positive effects are made autonomously and without human intervention in the shortest time and the resulting measures are implemented. The service provider and the customer must set up a structured and clear set of rules. The system then takes over all of the planning as well as the settlement of the orders. Maintenance personnel can concentrate on the operational process. The planners can deal with strategic issues and develop better consulting services.

\section{Technology}

Machine and order data.

All available data is processed and analyzed at all times.

\section{Organization}

Decisions are made by the system and they are available to the employee. All action is based on the planning of the system. Depending on the customer, the model and the contract design must be adapted. For safety reasons it is necessary that the dispatcher makes the decision and systemic liability issues are recorded in the contract. 


\section{Culture}

Transferring decision-making powers to digital software systems creates certain restrictions regarding the employee`s field of responsibility. Although the employee is the system designer as well as the inspector and exporter, he decides less autonomously than before because his decisions are system-oriented. Employees must therefore completely trust the systems, which guide and influence the decisions significantly.

\section{$5 \quad$ Critical Reflection and Outlook}

The described service maturity model is based on expert workshops as well as an in-depth literature review. In a first step, the maturity model describes the development stages of an industrial service provider on the way to becoming an agile, learning company. On this basis, one must define concrete recommendations for company measures, so that service enterprises can follow the path of maturity levels. For this purpose, it is necessary to disassemble the fields of action organization and culture in their dimensions and to assign the specific characteristics of the dimensions to the maturity levels. This is the only way to submit targeted recommendations for companies afterwards.

\section{References}

1. Herterich, M., Uebernickel, F., Brenner, W.: Industrielle Dienstleistungen 4.0. Springer, Wiesbaden (2016).

2. Martinsuo, M., Perminova-Harikoski, O., Turunen, T.: Strategic Change towards Future Industrial Service Business, https://tutcris.tut.fi/portal/files/4274023/strategic_change_towards_future_industrial_service_business.pdf, last accessed 2018/04/01.

3. Petry, T: Digital Leadership: Erfolgreiches Führen in Zeiten der Digital Economy. Haufe, Freiburg (2016).

4. Hermann, A., Löwer, P., Bohnenkamp, J., Stein, F.: Organigramm deutscher Unternehmen, http://assets.kienbaum.com/downloads/Organigramm-deutscher-Unternehmen_KienbaumStepstone-Studie_2017.pdf?mtime=20170427131752, last accessed 2018/04/01.

5. Baker, D., Kerry D.: The two faces of uncertainty avoidance: Attachment and adaptation. In: Journal of Behavioral and Applied Management 12(2), 128-142 (2011).

6. Ahlemann, F., Schroeder, C., Teuteberg, F.: Kompetenz-und Reifegradmodelle für das Projektmanagement. Grundlagen, Vergleich und Einsatz, https://www.researchgate.net/profile/Frank_Teuteberg2/publication/277323730_Kompetenz-_und_Reifegradmodelle_fur_das_Projektmanagement_Grundlagen_Vergleich_und_Einsatz/links/55678de308aeccd777378b12.pdf, last accessed 2018/04/01.

7. Schuh, G., Anderl, R., Gausemeier, J., Ten Hompel, M., Wahlster, W.: Industrie 4.0 Maturity Index: Die digitale Transformation von Unternehmen gestalten. Herbert Utz Verlag, München (2016).

8. Geissbauer, R., Griesmeier, A., Feldmann, S., Toepert, M.: Serviceinnovation: Potenziale industrieller Dienstleistungen erkennen und erfolgreich implementieren. Springer, Wiesbaden (2016).

9. Burger, T., Ganz, W., Pezzotta, G., \& Rapaccini, M.: Service development for product services: a maturity model and a field research. In: 2011 RESER Conference. Productivity of Services Next Gen-Beyond Output/Input. Hamburg, Germany, 7.-10. September 2011. Fraunhofer Verlag, Stuttgart (2011). 